\title{
Cyclically Repetition-free Words on Small Alphabets
}

\author{
Tero Harju \\ Department of Mathematics, University of Turku and Turku Centre for Computer Science \\ (TUCS), Finland \\ Dirk Nowotka ${ }^{1}$ \\ Institute for Formal Methods in Computer Science (FMI), Universität Stuttgart, Germany
}

\begin{abstract}
All sufficiently long binary words contain a square but there are infinite binary words having only the short squares 00,11 and 0101 . Recently it was shown by J. Currie that there exist cyclically square-free words in a ternary alphabet except for lengths $5,7,9,10,14$, and 17 . We consider binary words all conjugates of which contain only short squares. We show that the number $c(n)$ of these binary words of length $n$ grows unboundedly. In order for this, we show that there are morphisms that preserve circular square-free words in the ternary alphabet.
\end{abstract}

Key words: combinatorics on words, repetitions, conjugates, square-free words, cyclically square-free, almost square-free words

2000 MSC: 68R15

\section{Introduction}

We shall consider binary $\left(w \in\{0,1\}^{*}\right)$ and ternary $\left(w \in\{0,1,2\}^{*}\right)$ words. A word $u$ is a factor of a word $w$ if there are words $w_{1}$ and $w_{2}$ such that $w=w_{1} u w_{2}$. In this case, $u$ occurs in $w$. Two words $u$ and $v$ are conjugates if $u=x y$ and $v=y x$ for some words $x$ and $y$. The conjugacy class of a word $w$ consists of the words that are conjugates of $w$. For a given lexicographic order on words, the conjugacy class of any primitive word has a minimal element, which is called a Lyndon word. A nonempty factor $u^{2}(=u u)$ of a word $w$ is a square in $w$. The word $w$ is square-free if it has no squares. Moreover, $w$ is cyclically square-free if all of its conjugates are square-free.

While each binary word $w \in\{0,1\}^{*}$ of length at least four contains a square, R. Entringer, D. Jackson, and J. Schatz [3] showed that there exists an infinite word with only 5 different squares. Later A. Fraenkel and J. Simpson [4] showed

Email addresses: harju@utu.fi (Tero Harju), nowotka@fmi.uni-stuttgart.de (Dirk Nowotka)

${ }^{1}$ This work has been supported by the DAAD project D/08/08853 PPP Finland. 


\begin{tabular}{|c|c|c|c|c|c|c|c|c|c|c|c|c|c|c|}
\hline$n$ & 4 & 5 & 6 & 7 & 8 & 9 & 10 & 11 & 12 & 13 & 14 & 15 & 16 & 17 \\
\hline$c(n)$ & 3 & 2 & 2 & 2 & 1 & 0 & 0 & 0 & 3 & 0 & 1 & 0 & 0 & 0 \\
\hline
\end{tabular}

\begin{tabular}{|c|c|c|c|c|c|c|c|c|c|c|c|c|}
\hline$n$ & 18 & 19 & 20 & 21 & 22 & 23 & 24 & 25 & 26 & 27 & 28 & 29 \\
\hline$c(n)$ & 0 & 2 & 1 & 0 & 0 & 0 & 3 & 0 & 0 & 0 & 1 & 0 \\
\hline
\end{tabular}

\begin{tabular}{|c|c|c|c|c|c|c|c|}
\hline$n$ & 30 & 31 & 32 & 33 & 34 & 35 & 36 \\
\hline$c(n)$ & 1 & 0 & 0 & 0 & 0 & 0 & 2 \\
\hline
\end{tabular}

Table 1: Curious sequence of numbers of cyclically almost square-free binary words.

that there exists an infinite binary word having only the three squares 00,11 , and 0101 . We say that a binary word $w$ is almost square-free if its squares belong to the set $\{00,11,0101\}$ - but we do not allow the square 1010 .

Theorem 1 (Fraenkel-Simpson). For each $n \geq 1$, there exists an almost squarefree binary word of length $n$.

A simplified proof of Theorem 1 was given by N. Rampersad, J. Shallit, and M.-w. Wang [7] which was further shortened by the present authors in [5]. In this paper we consider cyclically words with short squares. The problem was motivated by the following result due to J. Currie [2].

Theorem 2 (Currie). There exists a cyclically square-free ternary word $w$ of length $n$ if and only if $n \notin\{5,7,9,10,14,17\}$.

A word $w$ is cyclically almost square-free if its conjugates are all almost square-free. We shall show in Theorem 8 that there are unboundedly long cyclically almost square-free binary words.

The exception list of lengths for cyclically almost square-free binary words is much more extensive than the list for cyclically square-free ternary words given by Currie. Indeed, it is an open problem to characterize the set $L_{\mathrm{cyc}}$ of lengths $n$ for which there exists a cyclically almost square-free binary word of length $n$. Also, even for each length $n \in L_{\text {cyc }}$ there seems to be only a small number of examples as seen from Table 1.

Let $c(n)$ denote the number of conjugacy classes of cyclically almost squarefree binary words of length $n$. Thus $c(n)$ equals the number of cyclically almost square-free binary Lyndon words having length $n$.

Remark 3. One can check that every almost square-free word $w$ (not necessarily cyclic) that omits either 000 or 111 as factors is not longer than 21. The longest such words are of length 21 :

$$
001000110010110001101 \text { and } 001000110010110001011
$$

and the variants obtained by renaming and reversal. Hence a Lyndon representative of a cyclically almost square-free binary word $w$ of length at least 22 starts with 11100 when the order is given as $1 \prec 0$. Indeed, it cannot start with 11101 
since it then has a conjugate starting with 0111011 which gives a contradiction at the next bit.

Example 4. Let us consider some examples of cyclically almost square-free binary words. We choose the ordering $1 \prec 0$ for the alphabet for our own convenience.

The Lyndon representatives of length $n=12$ are the following three words:

$$
\begin{aligned}
& 111001011000, \\
& 111000101100, \\
& 111000110010 .
\end{aligned}
$$

The Lyndon representatives of length $n=24$ are the following words:

$$
\begin{aligned}
& 111001011001110001011000, \\
& 111001011100011001011000, \\
& 111000110010111000101100 .
\end{aligned}
$$

There are, however, only two Lyndon representatives of length $n=36$ :

$$
\begin{aligned}
& 111001011001110001100101110001011000, \\
& 111001011100010110011100011001011000 \text {. }
\end{aligned}
$$

Despite of Table 1 suggesting that the number of cyclically almost square-free binary words decreases as the length grows, we will show

Theorem 5. The function $c(n)$ is unbounded:

$$
\limsup _{n \rightarrow \infty} c(n)=\infty \text {. }
$$

A mapping $\xi: X^{*} \rightarrow Y^{*}$ is called a morphism if $\xi(u v)=\xi(u) \xi(v)$, and $\xi$ is called a uniform morphism if additionally we have for some $k$ that $|\xi(a)|=k$ for all $a \in X$.

Now consider a uniform morphism $\xi:\{0,1,2\}^{*} \rightarrow\{0,1\}^{*}$ that takes cyclically square-free ternary words to cyclically almost square-free binary words. Such a morphism can be found by composing $\beta$ from Section 3 with $\alpha$ from Section 2 below, that is, $\xi(w)=\alpha(\beta(w))$. Note that this morphism is uniform since $|\beta(0)|_{i}=|\beta(1)|_{i}=|\beta(2)|_{i}$ for every $i \in\{0,1,2\}$ where $|w|_{a}$ denotes the number of occurrences of $a$ in $w$. Let $u$ and $v$ be two different cyclically square-free ternary words of the same length. Then $\xi(u)$ and $\xi(v)$ are two different cyclically almost square-free binary words of the same length. Hence, Theorem 5 follows from the next result. Let $c_{3}(n)$ denote the number of cyclically square-free ternary Lyndon words of length $n$ w.r.t. some fixed order.

Theorem 6. The function $c_{3}(n)$ is unbounded:

$$
\limsup _{n \rightarrow \infty} c_{3}(n)=\infty .
$$

This result will be proved in Section 3. We also state the following conjecture.

Conjecture 7. There exists an integer $N$ such that $c(n)>0$ for all $n \geq N$. 


\section{On Cyclically Binary Words with Short Squares}

The following theorem is proven in this section.

Theorem 8. There are unboundedly long cyclically almost square-free binary words.

Before we prove Theorem 8 let us recall a morphism from [5] that maps square-free ternary words to almost square-free binary words.

Let $\alpha:\{0,1,2\}^{*} \rightarrow\{0,1\}^{*}$ be the (nonuniform) morphism defined by

$$
\begin{aligned}
& \alpha(0)=A:=1^{3} 0^{3} 1^{2} 0^{2} 101^{2} 0^{3} 1^{3} 0^{2} 10, \\
& \alpha(1)=B:=1^{3} 0^{3} 101^{2} 0^{3} 1^{3} 0^{2} 101^{2} 0^{3} 10, \\
& \alpha(2)=C:=1^{3} 0^{3} 1^{2} 0^{2} 101^{2} 0^{3} 101^{3} 0^{2} 101^{2} 0^{2} .
\end{aligned}
$$

We notice in passing that these words are almost square-free, and the words $A$ and $C$ are cyclically almost square-free, but $B$ is not. Indeed, $B$ has a conjugate 100010111000101100011100101 with the long square $(10001011)^{2}$ as its prefix.

The following result was shown in [5].

Theorem 9. Let $w \in\{0,1,2\}^{*}$. Then $w$ is a square-free ternary word if and only if $\alpha(w)$ is an almost square-free binary word.

We now turn to the proof of the announced result.

Proof of Theorem 8. Let $w$ be a cyclically square-free ternary word provided by Theorem 2, and consider the binary word $\alpha(w)$. Assume that $|w| \geq 2$ w.l.o.g. By Theorem $9, \alpha(w)$ is almost square-free. The claim follows when $\alpha(w)$ is shown to be cyclically almost square-free. Assume, on the contrary, that $\alpha(w)$ has a conjugate $v$ that is not almost square-free. Without loss of generality, we can assume that $v$ has a square as a suffix, say

$$
v=s u^{2},
$$

where $u^{2}$ is a shortest possible square in the conjugates of $\alpha(w)$ with $u \notin\{0,1,01\}$. One easily checks that $|u| \geq 8$ by considering the words $\alpha(r)$ for $|r| \leq 2$ (see also the comment above Theorem 9). Since $w$ is cyclically square-free, it follows that $v \neq \alpha\left(w^{\prime}\right)$ for all conjugates $w^{\prime}$ of $w$.

Denote $\Delta=\{A, B, C\}$. We have the following marking property of $1^{3} 0^{3}$ :

$1^{3} 0^{3}$ occurs in cyclic words from $\Delta^{*}$ only as a prefix of $A, B$, or $C$.

Let $z$ be the shortest prefix of $v$, say $v=z t$, such that the conjugate $t z$ is in $\Delta^{*}$. In particular, there exists an $X \in \Delta$ such that $X=y z$ for some $y$.

Since $u^{2}$ is not a factor of the conjugate $t z$, we must have $|s|<|z|$, say $z=s z^{\prime}$. Therefore, $u^{2}=z^{\prime} t=z^{\prime} x^{\prime} y$ for some word $x^{\prime}$. However, the marking property and $|u| \geq 8$ and $|w| \geq 2$ imply $|u|>|y|$ and, hence,

$$
u=z^{\prime} x y \quad \text { and } \quad X=y s z^{\prime}
$$


for some prefix $x$ of a word in $\Delta^{*}$. Now $t z=x y z^{\prime} x y z \in \Delta^{*}$ which ends with the word $X=y z$. It follows that $x y z^{\prime} x \in \Delta^{*}$, i.e., $x$ occurs as a suffix and a prefix in $\Delta^{*}$. This implies that $x \in \Delta^{*}$ by the marking property. Hence also for the middle part $y z^{\prime} \in \Delta^{*}$. Since $y z^{\prime}$ is shorter than $X$, it follows that $y z^{\prime} \in \Delta$. Now both $y z^{\prime}$ and $y s z^{\prime}$ are in $\Delta$. This would imply that $|s|=3$ or 6 ; however there is no solution for these parameters in $\Delta$. (The length of the longest common prefix, rep. suffix, of two different words of $\Delta$ is 18 , resp. 4.)

\section{On the Number of Cyclically Square-Free Words}

A morphism is called (cyclically) square-free whenever the image of any (cyclically) square-free word is itself (cyclically) square-free. In this section we will construct a set of uniform cyclically square-free morphisms on $\{0,1,2\}^{*}$ such that an arbitrary number of cyclically square-free words of the same length can be generated.

We start from certain square-free factors taken from an infinite square-free word in order to find substitutions that preserve square-freeness. Then we introduce several markers that allow us to both ensure cyclically square-freeness and the construction of arbitrarily many different substitutions without sacrificing the preservation of square-freeness.

Thue gave in [8] the following morphism $\vartheta$ on $\{0,1,2\}^{*}$ which generates the infinite Thue word $\mathbf{t}$ when iterated starting in 0 . Consider

$$
\vartheta(0)=012, \quad \vartheta(1)=02, \quad \vartheta(2)=1
$$

which gives

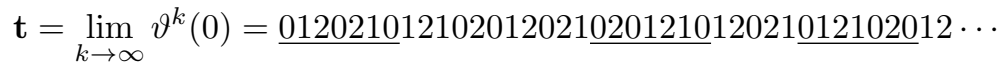

where we point out three underlined factors of $\mathbf{t}$ which will be used further below. It is well-known that $\mathbf{t}$ is square-free. The following morphism $\eta:\{0,1,2\}^{*} \rightarrow$ $\{0,1\}^{*}$ maps $\mathbf{t}$ to an overlap-free binary word [6], the so called Thue-Morse word,

$$
\eta(0)=011, \quad \eta(1)=01, \quad \eta(2)=0 .
$$

A word is called overlap-free if it has no overlapping factors, i.e., if no factor of the form awawa occurs where $a$ is a letter and $w$ is a (possibly empty) word. In particular the words in the following set do not occur in t:

$$
T_{\mathrm{no}}=\{010,212,1021,1201\} .
$$

Indeed, $\eta(010)=01101011$ which contains the overlap 10101. Assume that contrary to the claim 212 occurs in $\mathbf{t}$. Then it must be preceded and succeeded by 0 since $\mathbf{t}$ is square-free. But, $\eta(02120)=0110010011$ contains the overlap 1001001; a contradiction. If 1021 occurs in $\mathbf{t}$, then it must be preceded by 2 and succeeded by 0 by the previous arguments. But, then $\mathbf{t}$ contains the square 210210; a contradiction. A similar argument holds for the word 1201. 
So far, we have identified in $T_{\text {no }}$ square-free words that do not occur in $\mathbf{t}$. They will serve as markers in the proof of Theorem 6 below.

Iterating $\vartheta$ gives

$$
\begin{aligned}
\vartheta(0) & =012 \\
\vartheta^{2}(0) & =012021 \\
\vartheta^{3}(0) & =012021012102 \\
\vartheta^{4}(0) & =012021012102012021020121
\end{aligned}
$$

and

$$
\begin{aligned}
& \vartheta(1)=02 \\
& \vartheta(2)=1 \\
& \vartheta^{2}(1)=0121 \\
& \vartheta^{2}(2)=02 \\
& \vartheta^{3}(1)=01202102 \\
& \text { and } \quad \vartheta^{3}(2)=0121 \\
& \vartheta^{4}(1)=0120210121020121 \\
& \vartheta^{4}(2)=01202102
\end{aligned}
$$

Consider the words $\vartheta^{4}(0)$ and $\vartheta^{4}(1)$ and $\vartheta^{4}(2)$ that start with 012021 and that all have an occurrence in $\mathbf{t}$ followed by 0120 . Indeed, $\vartheta^{6}(0)$ is a prefix of $\mathbf{t}$ and $\vartheta^{6}(0)=\vartheta^{4}(012021)=\vartheta^{4}(0) \vartheta^{4}(1) \vartheta^{4}(2) \vartheta^{4}(0) \vartheta^{4}(2) \vartheta^{4}(1)$.

Let $\delta$ be a morphism on $\{0,1,2\}^{*}$ defined by

$$
\begin{aligned}
& \delta(0)=(012)^{-1} \vartheta^{4}(0) 012=021012102012021020121012, \\
& \delta(1)=(012)^{-1} \vartheta^{4}(1) 012=0210121020121012, \\
& \delta(2)=(012)^{-1} \vartheta^{4}(2) 012=02102012 .
\end{aligned}
$$

We have

Claim 10. The $\delta$-image of each factor of $\mathbf{t}$ occurs itself in $\mathbf{t}$ followed by 021.

Indeed, let $w$ be a factor of $\mathbf{t}$, then $\vartheta(w)$, and hence, $\vartheta^{4}(w)$ is a factor of $\mathbf{t}$. Therefore, $(012)^{-1} \vartheta^{4}(w)$ is a factor of $\mathbf{t}$ which proves the claim since $(012)^{-1} \vartheta^{4}(w a)$ occurs in $\mathbf{t}$, for some letter $a$ such that $w a$ occurs in $\mathbf{t}$, and 012021 is a prefix of $\vartheta^{4}(a)$.

Consider the factors 0201210 and 0120210 and 0121020 of $\mathbf{t}$ as marked in (1). Note that these factors are of the same length and have the same number of occurrences of 0,1 , and 2 , respectively.

Let us define the following uniform morphism $\beta$ on $\{0,1,2\}^{*}$ where the length of the images of letters is $|\beta(i)|=122$ :

$$
\begin{aligned}
& \beta(0)=\delta(0201210) 01, \\
& \beta(1)=\delta(0120210) 01, \\
& \beta(2)=\delta(0121020) 01 .
\end{aligned}
$$


Remark 11. We note that for different letters $a$ and $b$, the prefixes of length 61 and the suffixes of length 62 of $\beta(a)$ and $\beta(b)$ are different.

Claim 12. The images $\beta(i)$ are cyclically square-free for all $i \in\{0,1,2\}$.

Proof. The claim can be easily proven by inspection or a computer test. However, we give an alternative proof for illustrating some arguments also used later below.

By Claim 10 the prefix $\beta(i) 1^{-1}$ of $\beta(i)$ is a factor of $\mathbf{t}$ for all $i \in\{0,1,2\}$. The words $\beta(i)$ end with 1201 which is in the set $T_{\text {no }}$ of forbidden factors of $\mathbf{t}$. It follows that the words $\beta(i)$ are square-free. It is also straightforward to verify that $\beta(i)$ are cyclically square-free. Indeed, any cyclic square $x^{2}$ must contain the last letter 1 of $\beta(i)$. The case where $|x|<6$ is easily checked by hand. Note that $1 \beta(i) 1^{-1}$ begins with 1021 and $\beta(i)$ ends with 1201 . Hence, if $|x| \geq 6$ then $x$ contains 1021 or 1201 . But $1021,1201 \in T_{\text {no }}$ and therefore they occur at most once in any conjugate of $\beta(i)$ which contradicts that $x^{2}$ occurs in a conjugate of $\beta(i)$. This concludes the proof of Claim 12 .

Let $\pi$ be any permutation on $\{0,1,2\}$. We define the following morphisms

$$
\beta_{\pi}(i)=\beta(\pi(i))
$$

for $i \in\{0,1,2\}$. Before we show that every $\beta_{\pi}$ is cyclically square-free, we recall the following theorem by Thue [8]; see [1] for a slightly improved version.

Theorem 13. A morphism $\alpha$ is square-free if the following two conditions are satisfied:

(1) $\alpha(u)$ is square-free whenever $u$ is square-free with $|u| \leq 3$, and

(2) $\alpha(a)$ is not a proper factor of $\alpha(b)$ for any letters $a$ and $b$.

In order to show that the constructed morphisms are cyclically square-free we state the following result.

Proposition 14. A morphism $\alpha$ is cyclically square-free if the following two conditions are satisfied:

(1) $\alpha$ is square-free and

(2) $\alpha(a)$ is cyclically square-free for all letters a.

Proof. Let $w_{(i)}$ denote the $i$ th letter of the word $w$. Consider a cyclically squarefree word $w$ of length $n$ and suppose, contrary to the claim, that $\alpha(w)$ is not cyclically square-free. Let $x^{2}$ be a shortest square in a conjugate of $\alpha(w)$. Let $w^{\prime}=w_{(i)} w_{(i+1)} \cdots w_{(n)} w_{(1)} \cdots w_{(i-1)} w_{(i)}$. Then $x^{2}$ occurs in $\alpha\left(w^{\prime}\right)$ for some $i$. Now, $w^{\prime}$ is square-free if $w$ is cyclically square-free, except if $n=1$; a contradiction of either (1) or (2) in any case.

It is now straightforward to establish the cyclically square-freeness of any $\beta_{\pi}$ which implies Theorem 6 . 
Lemma 15. Let $\pi$ be any permutation on $\{0,1,2\}$. Then $\beta_{\pi}$ is a cyclically square-free morphism.

Proof. We begin by showing that $\beta_{\pi}$ is square-free. By Theorem 13 the squarefreeness of $\beta_{\pi}$ can be checked by hand. However, this is cumbersome and therefore we give an alternative proof avoiding Theorem 13. Suppose contrary to the claim that $\beta_{\pi}(w)$ contains a square $x^{2}$ where $w$ is square-free. Surely, $x^{2}$ does not occur in $\beta_{\pi}(a)$ for any letter $a$ by Claim 12 . Note that 1201021 occurs in $\beta_{\pi}(w)$ only at a point where two $\beta_{\pi}$ images of letters are concatenated. Assume that $|x| \geq 6$; the smaller cases can be easily excluded. Then, as in the proof of Claim 12, $x$ contains 1201 or 1021 . Both 1021 and 1201 mark the beginnings and ends of the $\beta_{\pi}$ images of letters, and hence, $\beta_{\pi}$ is injective. Let $u \in\{1021,1201\}$ be such that $u$ occurs in $x$. Suppose $u=1201$, the other case follows analogous reasons. Then either $u$ occurs in the beginning or end of $x$. We have then

$$
\begin{aligned}
x & =y u \beta_{\pi}\left(w_{(j)}\right) \beta_{\pi}\left(w_{(j+1)}\right) \cdots \beta_{\pi}\left(w_{(j+r)}\right) z \\
& =y u \beta_{\pi}\left(w_{(j+r+2)}\right) \beta_{\pi}\left(w_{(j+r+3)}\right) \cdots \beta_{\pi}\left(w_{(j+2 r+2)}\right) z,
\end{aligned}
$$

where $1<j<|w|-r$ and $-1 \leq r<|w| / 2$ and $z y u=\beta_{\pi}\left(w_{(j+r+1)}\right)$. Here the word $u$ is a marker and hence the $\beta_{\pi}$-images are aligned in the two occurrences of $x$, i.e., $w_{(j+\ell)}=w_{(j+r+2+\ell)}$ for $\ell=0, \ldots, r$. Now, $w_{(j-1)} \neq w_{(j+r+1)}$ and $w_{(j+2 r+3)} \neq w_{(j+r+1)}$, since $w$ is square free. However, $y u$ is a suffix of $\beta_{\pi}\left(w_{(j-1)}\right)$ and, by Remark 11, $|y u| \leq 61$. Also, $z$ is a prefix of $\beta_{\pi}\left(w_{(j+2 r+3)}\right)$ and thus $|z| \leq 60$. But now $|z y u| \leq 121$ gives a contradiction with $z y u=\beta_{\pi}\left(w_{(j+r+1)}\right)$. Therefore, $\beta_{\pi}$ is square-free. Claim 12 and Proposition 14 conclude the proof.

Now, Theorem 6 follows.

Theorem 6. The function $c_{3}(n)$ is unbounded:

$$
\limsup _{n \rightarrow \infty} c_{3}(n)=\infty \text {. }
$$

Proof. Indeed, the image of the cyclically square-free word 021 under $\beta_{\pi}$ gives a different cyclically square-free word for any permutation $\pi$ by Lemma 15 . Each of these cyclically square-free words starts with 021 , and hence, gives six new cyclically square-free words (one for each $\beta_{\pi}$ ). This process can be iterated arbitrarily many times. The uniformness of $\beta_{\pi}$ ensures that the images of a word are of the same length for each $\pi$. The number of different cyclically square-free words after $k$ iterations equals $6^{k}$ and they are of length $3 \cdot 122^{k}$.

Remark 16. We mention another approach to show Theorem 6 using substitutions instead of morphisms. Consider the following words of length 18:

$$
\begin{aligned}
u_{1} & =010201210201021012 \\
u_{2} & =010201210212021012 \\
v_{1} & =010201202120121012 \\
v_{2} & =010201202102010212 \\
w_{1} & =010201202101210212
\end{aligned}
$$


The substitution $s:\{0,1,2\}^{*} \rightarrow 2^{\{0,1,2\}^{*}}$ defined by

$$
s(0)=\left\{u_{1}, u_{2}\right\}, \quad s(1)=\left\{v_{1}, v_{2}\right\}, \quad s(2)=\left\{w_{1}\right\}
$$

preserves cyclically square-freeness, i.e., if $w$ is cyclically square-free, then so is each $u \in s(w)$. Now, the sequence $\left|s^{n}(0)\right|$ of elements in $s^{n}(0)$ is strictly increasing with increasing $n$, and thus proves our theorem.

This claim on $s$ needs to be verified in order to make this approach work. This can be shown by similar techniques as the ones used above in the proof of Lemma 15.

\section{Acknowledgement}

We would like to thank the anonymous referees for their careful work. Their comments greatly helped to improve this article.

\section{References}

[1] D. R. Bean, A. Ehrenfeucht, and G. F. McNulty. Avoidable patterns in strings of symbols. Pacific J. Math., 85(2):261-294, 1979.

[2] J. D. Currie. There are ternary circular square-free words of length $n$ for $n \geq 18$. Electron. J. Combin., 9(1):Note 10, 7 pp. (electronic), 2002.

[3] R. C. Entringer, D. E. Jackson, and J. A. Schatz. On nonrepetitive sequences. J. Combin. Theory, Ser. A, 16:159-164, 1974.

[4] A. S. Fraenkel and J. Simpson. How many squares must a binary sequence contain? Electronic J. Combin., 2(\#R2 ( electronic)), 1995.

[5] T. Harju and D. Nowotka. Binary words with few squares. Bull. EATCS, 89:164-166, 2006.

[6] M. Lothaire. Combinatorics on Words, volume 17 of Encyclopedia of Mathematics. Addison-Wesley, Reading, MA, 1983. Reprinted in the Cambridge Mathematical Library, Cambridge Univ. Press, 1997.

[7] N. Rampersad, J. Shallit, and M.-w. Wang. Avoiding large squares in infinite binary words. Theoret. Comput. Sci., 339:19-34, 2005.

[8] A. Thue. Über die gegenseitige Lage gleicher Teile gewisser Zeichenreihen. Norske Vid. Skrifter I. Mat.-Nat.Kl., Christiania, 1:1-67, 1912. 